\title{
Velocidade de caminhada como preditora da incapacidade funcional em idosos
}

Walking speed as predictor for functional disability in the elderly

\author{
Kelly Nascimento Lago \\ Universidade Estadual do Sudoeste da Bahia, Brasil
}

\section{Saulo Vasconcelos Rocha}

Universidade Estadual do Sudoeste da Babia, Brasil

svrocha@uesb.edu.br

Natália Silva Oliveira

Universidade Estadual do Sudoeste da Babia, Brasil

Tatiana Lima Brandäo

Universidade Estadual do Sudoeste da Babia, Brasil

Murillo Santos Souza

Universidade do Norte do Paraná, Brasil

Andree Phillipe Pimentel Coutinho

Universidade Federal de Santa Catarina, Brasil

\section{Resumo:}

O objetivo desse estudo foi analisar a velocidade de caminhada como preditora da incapacidade funcional (IF) em idosos. Tratase de um estudo epidemiológico, de corte transversal, realizado em um município do Estado da Bahia, região nordeste do Brasil com amostra probabilística de 310 idosos com média de idade de $71.62(\mathrm{DP}=8.15)$ anos, cadastrados nas Unidades de Saúde da Família do município. Foram construídas curvas Receiver Operating Characteristic (ROC) e determinado o critério discriminador da velocidade de caminhada de $2.44 \mathrm{~m}$ (segundos) para incapacidade funcional. Os tempos de caminhada que melhor discriminam a IF foram de $\geq 12,83$ segundos para os homens e $\geq 10,77$ segundos para mulheres. Conclui-se que a velocidade de caminhada tem boa capacidade de discriminar a IF em idosos e pode ser utilizada na avaliação gerontológica e em levantamento das condições de saúde dos idosos. Descritores: Atividade Física; Saúde do idoso; curvas ROC; Marcha.

Palavras-chave: Atividade Física, Saúde do idoso, curvas ROC, Marcha.

\section{Abstract:}

The objective of this study was to analyze walking speed as a predictor of functional disability (FD) in the elderly. This crosssectional, epidemiological study was carried out in a Municipality of the State of Bahia, in the Northeastern Region of Brazil, with a probabilistic sample of 310 elderly people with an average age of $71.62(\mathrm{SD}=8.15)$ years old, enrolled in the Family Health Units of the municipality. Receiver Operating Characteristic (ROC) curves were constructed and the criterion discriminating the walking speed of $2.44 \mathrm{~m}$ (seconds) for functional disability was determined. The walking times that best discriminated the FD were $\geq 12.83$ seconds for men and $\geq 10.77$ seconds for women. It was concluded that walking speed has a good ability to discriminate the FD in the elderly and it can be used in the gerontological evaluation and in the survey of the health conditions of the elderly. KEYWORDS: Exercise, Health of the elderly, ROC curves, Gait.

\section{INTRODUÇAO}

O envelhecimento é um processo de mudanças fisiológicas e psicológicas que reduzem a efetividade das funções do organismo e, aliado à falta de atividade física regular, compromete a capacidade funcional (Tribess, Virtuoso, 2005). 
Esse declínio funcional pode prejudicar as atividades de vida diária (AVD) repercutindo tanto nos aspectos individuais quanto sociais, fatores que influenciam a qualidade de vida (Ribeiro, Oliveira, Modena \& do Carmo Fonseca, 2002). Dessa forma, a capacidade funcional apresenta-se como um fator determinante na saúde do idoso, uma vez que a autonomia para realizar as tarefas cotidianas é superior à presença de patologias (Ramos, 2003).

Nesse contexto, a incapacidade funcional (IF) pode ser definida como a impossibilidade ou dificuldade do indivíduo para realizar tarefas cotidianas, indispensáveis para uma vida independente na comunidade (Parreira e Alves, 2014). Além disso, entre os fatores relacionados à capacidade para a realização das AVDs, a mobilidade apresenta-se como um requisito essencial no diagnóstico de incapacidade funcional em idosos.

A mobilidade refere-se à capacidade de deslocamento para realizar atividades do cotidiano e pode ser mensurada com enfoque gradual, desde atividades mais simples como mudanças de decúbito, transferência, levantar e sentar em uma cadeira, até as mais complexas que englobam, por exemplo, deambulação em distâncias determinadas e mudanças no curso da marcha, ou seja, atividades que exigem maior amplitude de movimento e resistência muscular (Bonardi, Souza, \& Moraes, 2007).

A avaliação da mobilidade do idoso pode contribuir para a formação de um perfil funcional, além de indicar a presença de patologias crônicas e o risco de dependência do idoso (Rogers, Rogers, Takeshima, \& Islam, 2003). Para tanto, o teste de velocidade de caminhada apresenta-se como um método de fácil acesso, baixo custo e reprodutibilidade satisfatória (Moreira, Oliveira, de Moura, Tapajós \& Maciel, 2013), apresentandose como um indicador importante da incapacidade funcional em idosos com renda mensal acima de um salário mínimo (Santos et al., 2014), porém ainda não está claro sua efetividade em idosos de baixa condição econômica.

A redução da velocidade de caminhada é recorrente no processo de envelhecimento e está relacionada a fatores adversos à saúde como acidente vascular encefálico, quedas e fratura de quadril, além de ser influenciada pelo desempenho cognitivo (Lenardt et al., 2015; Peel, Kuys, e Klein, 2013). Ainda, os escores de desempenho em testes de velocidade da caminhada parece estar relacionada à redução do desempenho nas atividades instrumentais da vida diária. Estima-se que a cada $0,10 \mathrm{~m} / \mathrm{s}$ de decréscimo na velocidade da caminhada, ocorre uma redução de 10\% na capacidade de realização dessas atividades (Lopopolo, Greco, Sullivan, Craik, \& Mangione, 2006).

Diante do exposto, torna-se importante uma maior compreensão do desempenho de idosos em testes de velocidade de caminhada em diferentes contextos do envelhecimento humano e suas repercussões na IF no intuito de ampliar o número de evidências acerca da relação entre essas variáveis. Para tanto, o objetivo desse estudo foi analisar a velocidade de caminhada como preditora da IF em idosos.

\section{Metodologia}

Trata-se de um estudo de corte transversal, realizado num município de Ibicuí, localizado no interior do sudoeste da Bahia em fevereiro de 2014.

A pesquisa foi avaliada e aprovada pelo Comitê de Ética e Pesquisa da Universidade Estadual do Sudoeste da Bahia (CAAE: 22969013.0.0000.0055), de acordo com os princípios éticos presentes na Resolução $n^{\circ}$ 466/2012 (Brasil, 2012) do Conselho Nacional de Saúde.

\section{Amostra}

A amostra foi composta por indivíduos com 60 anos de idade ou mais, cadastrados na Estratégia de Saúde da Família (ESF) do município de Ibicuí-BA. Para definir o tamanho da amostra foram utilizados os critérios propostos por Luiz e Magnanini (2000) para populações finitas, sendo adotados: nível de significância de 5\%, intervalo de confiança de $95 \%$ e erro tolerável de 3 p.p. Foram incluídos $10 \%$ a mais de 
sujeitos devido a possibilidade de perdas e recusas e excluídos os indivíduos com diagnóstico de demência ou qualquer outro tipo de alteração cognitiva (registrada nos prontuários médicos) que pudesse comprometer a veracidade das informações fornecidas e os que não realizaram a avaliação do teste de velocidade de caminhada. Após contabilização das perdas e recusas, a amostra final foi de 310 idosos, sendo 201 da zona urbana e 109 da zona rural. A taxa de respostas foi de $91,2 \%$, com $8,8 \%(n=31)$ de recusas e $9,2 \%(\mathrm{n}=29)$ de exclusão (Figura 1$)$.

FIGURA 1

Organograma de distribuição da amostra do estudo.

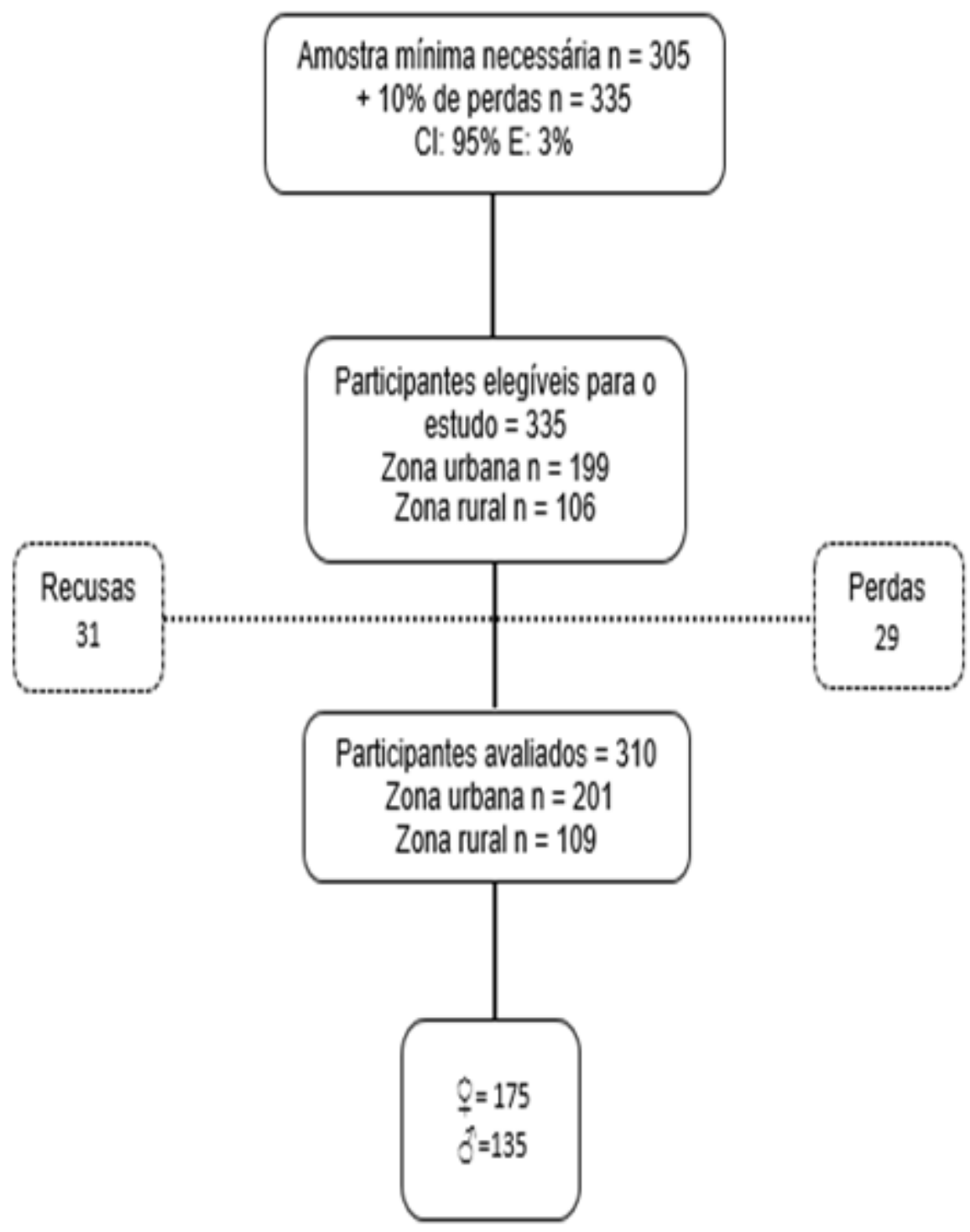

\section{Procedimentos}

Para a coleta de dados, utilizou-se o Instrumento de Avaliação da Saúde de Idosos (IASI), um questionário multidimensional construído a partir de outros instrumentos padronizados e validado por Pedreira e colaboradores (2016). O IASI é composto por sete blocos que objetivam avaliar diversas questões relacionadas à saúde do idoso, como informações pessoais e sociodemográficas, nível de atividade física e capacidade funcional. 
Foram incluídos no presente estudo informações sociodemográficas: sexo (masculino/feminino), idade (anos), escolaridade (alfabetizados e não alfabetizados) e renda familiar (em reais).

Para medir o índice de massa corporal (IMC) utilizou-se a fórmula peso $(\mathrm{kg}) /$ altura $\left(\mathrm{m}^{2}\right)$. Os pontos de cortes adotados foram propostos por Lipschitz (1994): baixo peso $<22 \mathrm{~kg} / \mathrm{m}^{2}$, peso normal $22-27 \mathrm{~kg} / \mathrm{m}^{2}$ e sobrepeso/obesidade $>27 \mathrm{~kg} / \mathrm{m}^{2}$.

A inatividade física no lazer foi avaliada por meio de questão dicotômica: sim (não participa de no mínimo 150 minutos por semana de atividades físicas moderadas/intensas no seu tempo livre) e não (participa de no mínimo 150 minutos de atividade física moderada/intensa por semana no tempo livre).

Para avaliar a velocidade de caminhada (tempo dispendido medido em segundos), utilizou-se o teste de caminhada de 2,44m (Rikli e Jones, 2008). Esse teste tem o objetivo de estimar características espaciais e temporais da marcha (Watson, 2002). Foi solicitado aos idosos que caminhassem em sua velocidade usual o trajeto de 2,44m demarcado por uma cadeira e um cone. O participante foi instruído a: levantar da cadeira, ir até o cone e retornar à cadeira. $\mathrm{O}$ teste foi realizado três vezes e o que obteve melhor desempenho considerado para análise de dados.

A incapacidade funcional foi analisada pela escala de Lawton e Brody (1969) que avalia as atividades instrumentais de vida diária (AIVD), que são: atividades de utilizar o telefone, meio de transporte para lugares distantes, realizar compras, preparar a refeição, limpar e arrumar a casa, consumo de medicamentos na hora certa e administrar as próprias finanças (Lopes dos Santos e Virturoso Júnior, 2008). Foram considerados dependentes aqueles que acumularam até 25 pontos na escala (Lawton e Brody, 1969).

\section{Tratamento estatístico}

Os dados foram tabulados pelo software Epidata e analisados no programa estatístico Medcalc, v 11.4.4 e no Statistical Package for Social Sciences (SPSS), versão 22.0. Na análise dos dados foram utilizados procedimentos da estatística descritiva e as curvas Receiver Operating Characteristic (ROC) para identificar o poder discriminatório e os pontos de corte do teste de velocidade da caminhada para identificar a IF.

\section{Resultados E Discuss Ão}

A média de idade dos entrevistados foi 71.62 anos $(\mathrm{DP}=8.15)$ sendo a maioria do sexo feminino (56,5\%). Maioria era alfabetizada e a renda média mensal foi de 708,26 reais ( $\mathrm{DP}=303.69)$. Quanto aos hábitos de vida, 69\% afirmaram não realizar atividade física no lazer e 38,4\% apresentaram sobrepeso/ obesidade. A prevalência de comprometimento cognitivo foi de 18,7\% (Tabela1). 


\section{TABELA 1}

\begin{tabular}{|l|l|l|}
\hline Variável & N & $\%$ \\
\hline SEXO & 175 & 56,5 \\
Feminino & 135 & 43,5 \\
Masculino & & \\
\hline ESCOLARIDADE & 174 & 56,1 \\
Alfabetizado & 136 & 43,9 \\
Não alfabetizado & & \\
\hline IF NO LAZER & 96 & 31,0 \\
Não & 214 & 69,0 \\
Sim & & \\
\hline IMC & 119 & 38,4 \\
Sobrepeso & 117 & 37,7 \\
Peso normal & 64 & 20,6 \\
Baixo peso & & \\
\hline DÉFICIT COGNITIVO & & 81,3 \\
Não & 58 & 18,7 \\
Sim & & \\
\hline AIVD & 149 & 48,1 \\
Independente & 161 & 51,9 \\
Dependente & & \\
\hline
\end{tabular}

Fonte: IF: Inatividade Física; IMC: Índice de Massa Corporal; AIVD: Atividade Instrumental de Vida Diária.

No teste de velocidade da caminhada observou-se que o tempo médio de realização do teste foi de 9,0 $(\mathrm{DP}=3.68)$ segundos. A prevalência geral de IF foi de $51,9 \%(n=161)$.

A análise das áreas sob a curva ROC mostrou que os valores foram de 0.79 (IC: 95\% 0,62 - 0,76) para mulheres e 0,91 (IC: 95\% 0,85-0,95) para os homens (Figuras 02 e 03). 
FIGURA 2

Área sob a curva ROC entre a velocidade de caminhada e a incapacidade funcional do sexo feminino

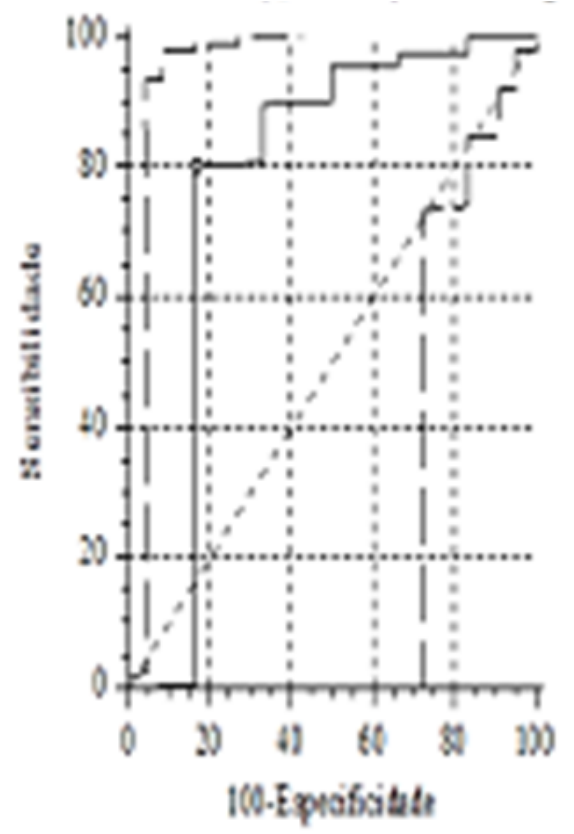

FIGURA 3

Área sob a curva ROC entre a velocidade de caminhada e a incapacidade funcional do sexo masculino.

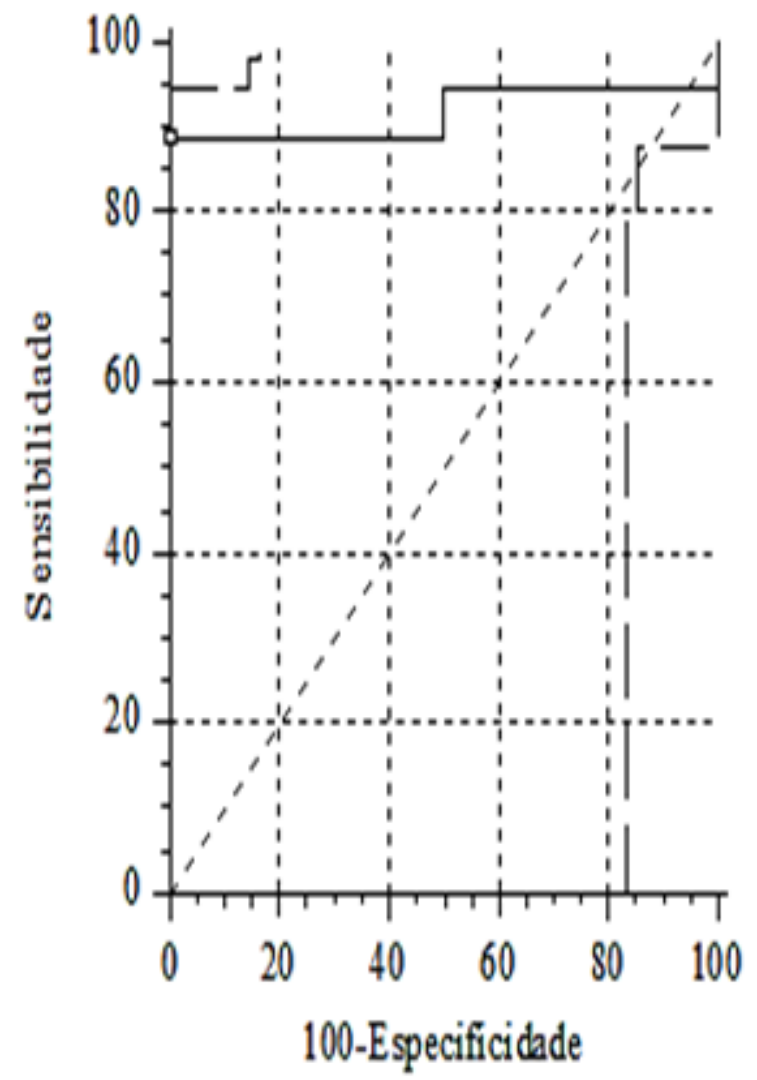


Os pontos de corte do desempenho no teste de velocidade de caminhada que melhor discriminaram a IF foram de 12.83 segundos ou mais e 10.77 segundos ou mais entre homens e mulheres respectivamente. (Tabela 2).

TABELA 2

Pontos de corte da velocidade de caminhada como discriminadores para incapacidade funcional.

\begin{tabular}{|l|l|l|l|}
\hline & Pontos de Corte* & Sensibilidade(\%) & Especificidade (\%) \\
\hline Geral & $>10,87$ & 80,1 & 83,3 \\
\hline Mulheres & $>10,77$ & 79,3 & 75,0 \\
\hline Homens & $>12,83$ & 88,6 & 100,00 \\
\hline
\end{tabular}

* Teste de caminhada $(2,44 \mathrm{~m})$ escore em segundos

A análise da área sob a curva mostrou que o teste de velocidade de caminhada tem uma boa capacidade preditiva da IF em idosos, esses achados estão em concordância com o estudo realizado por Santos et.al. (2014), o único estudo encontrado que fez uma análise semelhante, com idosos residentes no município de Uberaba-MG.

A avaliação da velocidade da caminhada é uma importante medida do desempenho global, pois mensura a capacidade energética, força e resistência muscular, equilíbrio dinâmico e a mobilidade (Studenski et al., 2011). A avaliação por meio desse indicador faz-se importante, tendo em vista que a redução da velocidade de caminhada em idosos têm demonstrado relação com a capacidade de controle e equilíbrio do corpo e consequentemente, maior incidência de quedas (Montero-Odasso et al., 2005), o que compromete a capacidade funcional e a qualidade de vida dessa população.

Com o envelhecimento, a redução da velocidade de caminhada ocorre devido às alterações estruturais e funcionais decorrentes desse processo, que podem gerar adaptações as modificações neuromusculares e do sistema musculoesquelético, que consequentemente podem diminuir os valores de força (Riley, Della Croce, \& Kerrigan, 2001).

Os resultados do presente estudo mostraram que os melhores pontos de corte (os que apresentaram melhores equilíbrios entre sensibilidade e especificidade) foram de $\geq 12,83 \mathrm{e} \geq 10,77$ segundos entre homens e mulheres respectivamente. Esses escores foram superiores aos encontrados por Santos et al. (2014) (> 2,57 para homens e $>3,19$ segundos para mulheres), demonstrando que a heterogeneidade do processo de envelhecimento exerce impacto significativo com relação aos parâmetros diagnósticos utilizados na avaliação gerontologica.

Além disso, identificou-se que o tempo no teste de caminhada para discriminar a IF nos homens foi superior ao das mulheres. Melhores desempenhos em testes de aptidão física entre os homens (Pinheiro, Passos, Coqueiro, Fernandes, \& Barbosa, 2013) e no teste de velocidade de caminhada de 2,44m (Rossi e Sader, 2008) também foram observados em outros estudos.

As mulheres estão mais susceptíveis a diminuição da secção transversal dos músculos, menor densidade muscular, maior quantidade de conteúdo gorduroso intramuscular (Rossi e Sader, 2008) e de exposição a doenças crônicas (Barbosa, Souza, Lebrão, Laurenti, \& Marucci, 2005), o que pode favorecer as limitações funcionais (Barbosa et al., 2005). 
Em outra mão, os homens apresentam melhor vantagem no teste de velocidade de caminhada, pois o desempenho neste teste está atrelado a amplitude dos passos, que depende da estatura e potência muscular dos membros inferiores (Gonzaga, Barros, Lisboa, Barbieri, \& Gobbi, 2011).

Este estudo apresenta algumas limitações que devem ser apontadas. A avaliação da incapacidade funcional por meio do auto-relato pode subestimar algumas informações e é passível de viés de memória. O teste de velocidade de caminhada pode ser influenciado por aspectos motivacionais.

Por outro lado, o estudo analisou uma amostra representativa de uma região do país ainda pouco investigada, utilizando instrumentos válidos de medida, oferecendo assim informações importantes para que pesquisadores gestores e profissionais de saúde possam desenvolver ações de cuidado mais direcionadas a realidade dessa população.

\section{Conclusão}

Os resultados do presente estudo indicaram que a velocidade de caminhada do teste de $2,44 \mathrm{~m}$ tem uma boa capacidade de discriminar a incapacidade funcional em idosos. Recomenda-se a utilização do teste de velocidade de caminhada em ações no âmbito da clínica, avaliação gerontológica e levantamentos com propósito de avaliar as condições de saúde de idosos.

\section{REFERÊNCIAS}

Alves, L. C., Leite, I. D. C., \& Machado, C. J. (2008). Conceituando e mensurando a incapacidade funcional da população idosa: uma revisão de literatura. Ciência \& Saúde Coletiva, 13, 1199-1207.

Barbosa, A. R., Souza, J. M., Lebrão, M. L., Laurenti, R., \& Marucci, M. D. F. N. (2005). Functional limitations of Brazilian elderly by age and gender differences: data from SABE Survey. Cadernos de Saúde Pública, 21(4), 1177-1185. doi: https://doi.org/10.1590/S0102-311X2005000400020

Bonardi, G., Souza, V. B., \& Moraes, J. F. D. (2007). Incapacidade funcional e idosos: um desafio para os profissionais. Scientia Medica, 17(3), 138-144.

Gonzaga, J. D. M., Barros, S. E. B., Lisboa, M. G. D. C., Barbieri, F. A., \& Gobbi, L. T. B. (2011). Efeitos de diferentes tipos de exercício nos parâmetros do andar de idosas. Revista Brasileira de Medicina do Esporte, 166-170.

Lawton, M.P., \& Brody, E.M. (1969). Avaliação de pessoas idosas: atividades auto-sustentadas e instrumentais da vida diária. Ogerontologista, 9(3_Part_1), 179-186.

Lenardt, M. H., Sousa, J. A. V. D., Grden, C. R. B., Betiolli, S. E., Carneiro, N. H. K., \& Ribeiro, D. K. D. M. N. (2015). Gait speed and cognitive score in elderly users of the primary care service. Revista brasileira de enfermagem, 68(6), 1163-1168.

Lipschitz, D.A. (1994). Rastreamento do estado nutricional em idosos. Atenção Primária, 21(1), 55-67.

Lopes dos Santos, R., \& Virtuoso Júnior, J. S. (2008). Confiabilidade da versão brasileira da escala de atividades instrumentais da vida diária. Revista Brasileira em Promoção da Saúde, 21(4). doi: https://doi.org/10.5020/18 061230.2008.p290

Lopopolo, R. B., Greco, M., Sullivan, D., Craik, R. L., \& Mangione, K. K. (2006). Effect of therapeutic exercise on gait speed in community-dwelling elderly people: a meta-analysis. Physical Therapy, 86(4), 520-540. doi: https ://doi.org/10.1093/ptj/86.4.520

Luiz, R. R., \& Magnanini, M. M. (2000). A lógica da determinaçäo do tamanho da amostra em investigaçöes epidemiológicas. Cad. saúde colet., (Rio J.), 8(2), 9-28.

Montero-Odasso, M., Schapira, M., Soriano, E. R., Varela, M., Kaplan, R., Camera, L. A., \& Mayorga, L. M. (2005). Gait velocity as a single predictor of adverse events in healthy seniors aged 75 years and older. The Journals of Gerontology Series A: Biological Sciences and Medical Sciences, 60(10), 1304-1309. doi: https://doi.org/10.1093 /gerona/60.10.1304 
Moreira, M. A., Oliveira, B. S., de Moura, K. Q., Tapajós, D. M., \& Maciel, Á. C. C. (2013). A velocidade da marcha pode identificar idosos com medo de cair?. Revista Brasileira de Geriatria e Gerontologia, 16(1), 71-80.

Pedreira, S., Borges, R., Vasconcelos Rocha, S., dos Santos, C. A., Carneiro Vasconcelos, L. R., \& Cerqueira Reis, M. (2016). Validade de conteúdo do Instrumento de Avaliação da Saúde do Idoso. Einstein (16794508), 14(2). doi: https://doi.org/10.1590/S1679-45082016AO3455

Peel, N.M., Kuys, S.S. e Klein, K. (2013). Velocidade de marcha como medida na avaliação geriátrica em ambientes clínicos: uma revisão sistemática. Os diários da gerontologia: Série A, 68(1), 39-46. doi: https://doi.org/10.10 93/gerona/gls 174

Pinheiro, P. A., Passos, T. D., Coqueiro, R. D. S., Fernandes, M. H., \& Barbosa, A. R. (2013). Motor performance of the elderly in northeast Brazil: differences with age and sex. Revista da Escola de Enfermagem da USP, 47(1), 128-136. doi: https://doi.org/10.1590/S0080-62342013000100016

Ramos, L. R. (2003). Fatores determinantes do envelhecimento saudável em idosos residentes em centro urbano: Projeto Epidoso, São Paulo. Cadernos de Saúde Pública, 19, 793-797.

Ribeiro, R. D. C. L., Oliveira, A. I., Modena, C. M., \& do Carmo Fonseca, M. (2002). Capacidade funcional e qualidade de vida de idosos. Estudos Interdisciplinares sobre o Envelhecimento, 4.

Rikli, R. E., \& Jones, J. C. (2008). Teste de Aptidão Física para Idosos. Human Kinetics.(Tradução de Sonia Regina de Castro Bidutte), pp. 66-98.

Riley, P. O., Della Croce, U., \& Kerrigan, D. C. (2001). Effect of age on lower extremity joint moment contributions to gait speed. Gait \& posture, 14(3), 264-270. doi: https://doi.org/10.1016/S0966-6362(01)00133-3

Rogers, M. E., Rogers, N. L., Takeshima, N., \& Islam, M. M. (2003). Methods to assess and improve the physical parameters associated with fall risk in older adults. Preventive medicine, 36(3), 255-264. doi: https://doi.org/1 $0.1016 /$ S0091-7435(02)00028-2

Rossi, E., \& Sader, C. S. (2008). Envelhecimento do sistema osteoarticular. Einstein, 6(1), S7-12.

Santos, A. S. D., Tribess, S., Pinto, L. L. T., Ribeiro, M. D. C. L., Rocha, S. V., \& Júnior, J. S. V. (2014). Velocidade de caminhada como indicador para a incapacidade funcional em idosos. Motricidade, 10(3), 50-60. doi: https:/ /doi.org/10.6063/motricidade.10(3).3186

Studenski, S., Perera, S., Patel, K., Rosano, C., Faulkner, K., Inzitari, M., ... \& Nevitt, M. (2011). Gait speed and survival in older adults. Jama, 305(1), 50-58. doi:https://doi.org/10.1001/jama.2010.1923

Tribess, S., \& Virtuoso Jr, J. S. (2005). Prescrição de exercícios físicos para idosos. Revista saude. com, 1(2), 163-172.

Watson, M. J. (2002). Refining the ten-metre walking test for use with neurologically impaired people. Physiotherapy, 88(7), 386-397. doi: https://doi.org/10.1016/S0031-9406(05)61264-3 